Geometry ${ }_{6} \mathcal{T}$ Topology

Volume 4 (2000) 293-307

Published: 10 October 2000

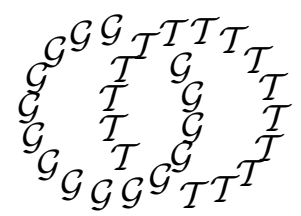

\title{
Normal all pseudo-Anosov subgroups of mapping class groups
}

\author{
Kim WhitTLESEY \\ Department of Mathematics, The Ohio State University \\ $231 \mathrm{~W}$ 18th Avenue, Columbus, OH 43210, USA \\ Email: whittle@math.ohio-state.edu
}

\begin{abstract}
We construct the first known examples of nontrivial, normal, all pseudo-Anosov subgroups of mapping class groups of surfaces. Specifically, we construct such subgroups for the closed genus two surface and for the sphere with five or more punctures. Using the branched covering of the genus two surface over the sphere and results of Birman and Hilden, we prove that a reducible mapping class of the genus two surface projects to a reducible mapping class on the sphere with six punctures. The construction introduces "Brunnian" mapping classes of the sphere, which are analogous to Brunnian links.
\end{abstract}

AMS Classification numbers Primary: 57M60

Secondary: 20F36, 57N05

Keywords: Mapping class group, pseudo-Anosov, Brunnian

Proposed: Joan Birman

Seconded: Shigeyuki Morita, Walter Neumann
Received: 24 November 1999

Revised: 28 September 2000

Copyright Geometry and Topology 


\section{Introduction}

In 1985, R C Penner, D D Long, and J D McCarthy asked the following question [7]: does there exist a nontrivial, normal subgroup of the mapping class group of a surface, all of whose nontrivial elements are pseudo-Anosov? This paper gives a positive answer for the case of a closed genus two surface and for the case of the sphere with five or more punctures. The approach is to construct a normal subgroup that avoids reducible and periodic elements; by work of Thurston, the remaining elements must all be pseudo-Anosov.

A Brunnian link is defined to be a nontrivial link such that every proper sublink is trivial. In section two we will define "Brunnian" mapping classes of the sphere and "Brunnian" sphere braids. Roughly speaking, a Brunnian sphere braid is analogous to a Brunnian link: it cannot "lose" a strand without becoming trivial; similarly, "Brunnian mapping classes of the n-punctured sphere" are those that become trivial if any of the punctures is "forgotten." The subgroup consisting of Brunnian mapping classes is a normal subgroup of the mapping class group of the n-punctured sphere. In section four we show that for $n \geq 5$, this subgroup has no reducible or periodic elements, thus proving

Theorem 1.1 For $n \geq 5$, The Brunnian subgroup $\operatorname{Br}\left(S^{2}, n\right)$ is a nontrivial subgroup of $M\left(S^{2}, n\right)$ all of whose nontrivial elements are pseudo-Anosov.

We use work of Birman and Hilden to lift the Brunnian subgroup of the mapping class group of the 6-punctured sphere to a "Brunnian subgroup" of the mapping class group of the closed genus two surface. We prove in section five that if a genus two mapping class is reducible, then the projection to the punctured sphere must also be reducible. The Brunnian subgroup for the genus two surface thus has no reducible elements except for the involution that gives the branched covering. To get rid of this element, we intersect with the kernel of the usual $\mathbb{Z}_{3}$ homology representation.

Theorem 1.2 The intersection of the Brunnian subgroup $\operatorname{Br}\left(\Sigma_{2}\right)$ and the kernel of the $\mathbb{Z}_{3}$ homology representation, $\rho: M\left(\Sigma_{2}\right) \rightarrow S p\left(4, \mathbb{Z}_{3}\right)$, is a nontrivial normal subgroup of $M\left(\Sigma_{2}\right)$, all of whose nontrivial elements are pseudo-Anosov.

\section{Background and definitions}

Let $Y$ be an orientable surface, possibly with boundary, and let $\left\{y_{1}, \ldots, y_{n}\right\}$ be an $n$-element subset of $Y$. We begin by introducing a notation that will let us easily distinguish between several different mapping class groups for $Y$. 
Definition 2.1 We define

$F(Y, n)$ to be the group of all orientation preserving homeomorphisms $h: Y \rightarrow Y$ that induce the identity permutation on the set of boundary components of $Y$ and take the set of points $\left\{y_{1}, \ldots, y_{n}\right\}$ to itself, and

$M(Y, n)=\pi_{0}(F(Y, n))$ to be the corresponding mapping class group,

$F^{\partial}(Y, n)$, with a superscript $\partial$, to be the group of all orientation preserving homeomorphisms $h: Y \rightarrow Y$ which fix the boundary pointwise and take the set of points $\left\{y_{1}, \ldots, y_{n}\right\}$ to itself, and $M^{\partial}(Y, n)=\pi_{0}\left(F^{\partial}(Y, n)\right.$ to be the corresponding mapping class group,

$F_{p}(Y, n)$, with a subscript $p$ (in analogy with pure braids), to be the group of all orientation preserving homeomorphisms $h: Y \rightarrow Y$ that induce the identity permutation on the set of boundary components of $Y$, and also fix the $y_{i}$ pointwise, ie, $h\left(y_{i}\right)=y_{i}$ for all $1 \leq i \leq n$,

$M_{p}(Y, n)=\pi_{0}\left(F_{p}(Y, n)\right)$ to be the corresponding mapping class group,

$F_{p}^{\partial}(Y, n)$, with both a superscript $\partial$ and a subscript $p$, to be the group of all orientation preserving homeomorphisms $h: Y \rightarrow Y$ which fix the boundary pointwise and fix the $y_{i}$ pointwise, and $M_{p}^{\partial}(Y, n)=\pi_{0}\left(F_{p}^{\partial}(Y, n)\right)$ to be the corresponding mapping class group.

Note that the elements of these mapping class groups are not permitted to permute the boundary curves. A superscript $\partial$ denotes that the homeomorphisms and isotopies restrict to the identity on the boundary curves. A subscript $p$ denotes that the homeomorphisms do not permute the points $y_{1}, \ldots, y_{n}$. If the surface $Y$ has no boundary, then $M^{\partial}(Y, n)=M(Y, n)$; if there are no specified points, then we write simply $M(Y)$ or $M^{\partial}(Y)$. The natural homomorphism from $M^{\partial}(Y, n)$ to $M(Y, n)$ has kernel generated by Dehn twists along curves parallel to the boundary curves.

The mapping class group most commonly found in the literature would have $M(Y)$ as a subgroup; in these larger mapping class groups, mapping classes 
may permute the boundary components, and isotopies do not restrict to the identity on the boundary components.

The notation $[f]$ is used to denote the mapping class represented by a member $f$ of any of these homeomorphism groups.

Theorem 2.1 (Thurston [4]) If $Y$ is an orientable surface of negative Euler characteristic, then a mapping class $[h]$ of $M(Y)$ must be either

(i) periodic, ie, $[h]^{n}=1$ for some $n \neq 0$, or

(ii) reducible, ie, having a representative that leaves invariant a non-empty collection of disjoint essential simple closed curves, none of which are isotopic to boundary curves, or

(iii) pseudo-Anosov, ie, having a representative that leaves invariant a transverse pair of singular foliations $F^{s}, F^{u}$ with transverse measures $\mu^{s}, \mu^{u}$ such that $h\left(F^{s}, \mu^{s}\right)=\left(F^{s},(1 / \lambda) \mu^{s}\right)$ and $h\left(F^{u}, \mu^{u}\right)=\left(F^{u}, \lambda \mu^{u}\right)$ for some $\lambda>1$.

In knot theory, a Brunnian link is defined to be a nontrivial link such that every proper sublink is trivial [8, page 67]. In [6] Levinson introduces disk braids with an analogous property: a "decomposable braid" is one such that if any single arbitrary strand is removed, the remaining braid is trivial. We will define a Brunnian sphere braid analogously: removal of any strand gives a trivial braid. See Figure 1. Note that these braids must always be pure. Similarly, a Brunnian mapping class of the sphere will be one where if we "forget" any of the points, there is an isotopy to the identity fixing all of the other points. Note that in order to "remove" a strand or "forget" a point in a well defined way, we need to assume that the permutation induced by the mapping class actually fixes that strand or point.

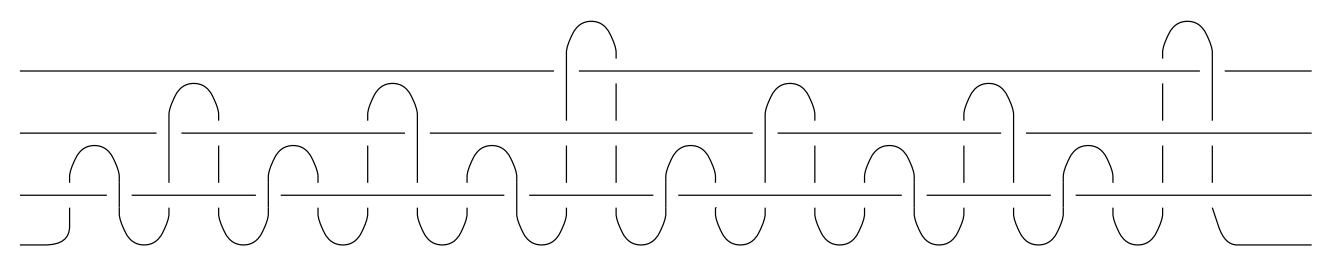

Figure 1: A Brunnian sphere braid with four strands

Formally, then, let

$B\left(S^{2}, n, y_{i}\right)$ be the subgroup of $B\left(S^{2}, n\right)$ consisting of homeomorphisms such that $h\left(y_{i}\right)=y_{i}$, ie, the induced permutation of the points leaves $y_{i}$ fixed, and let 


$$
M\left(S^{2}, n, y_{i}\right)=\pi_{0}\left(B\left(S^{2}, n, y_{i}\right)\right) .
$$

Note that $M\left(S^{2}, n, y_{i}\right)$ is a subgroup of $M\left(S^{2}, n\right)$ of index $n$. Since a homeomorphism in $B\left(S^{2}, n, y_{i}\right)$ leaves the set of $n-1$ points $\left\{y_{1}, \ldots, y_{n}\right\} \backslash y_{i}$ invariant, there is a well defined inclusion

$$
B\left(S^{2}, n, y_{i}\right) \rightarrow B\left(S^{2}, n-1\right),
$$

which induces a homomorphism

$$
L_{i}^{\prime}: M\left(S^{2}, n, y_{i}\right) \rightarrow M\left(S^{2}, n-1\right) .
$$

We now consider the intersection of all $n$ of these kernels, $\bigcap_{i=1}^{n} \operatorname{ker}\left(L_{i}^{\prime}\right)$. This is clearly a normal subgroup of $M\left(S^{2}, n\right)$. An element in this subgroup must act as the identity permutation on the $n$ points.

Alternatively, if we define $L_{i}$ to be the restriction of $L_{i}^{\prime}$ to $M_{p}\left(S^{2}, n\right)$,

$$
L_{i}: M_{p}\left(S^{2}, n\right) \rightarrow M_{p}\left(S^{2}, n-1\right),
$$

then

$$
\bigcap_{i=1}^{n} \operatorname{ker}\left(L_{i}\right)=\bigcap_{i=1}^{n} \operatorname{ker}\left(L_{i}^{\prime}\right) .
$$

Definition 2.2 We will call the maps $L_{i}$ the forget $y_{i}$ maps, and define the $n$-strand Brunnian subgroup of the sphere, $\operatorname{Br}\left(S^{2}, n\right)$, to be

$$
\operatorname{Br}\left(S^{2}, n\right)=\bigcap_{i=1}^{n} \operatorname{ker}\left(L_{i}\right)=\bigcap_{i=1}^{n} \operatorname{ker}\left(L_{i}^{\prime}\right) .
$$

Elements of this subgroup will be called Brunnian mapping classes of $M\left(S^{2}, n\right)$.

We now define Brunnian mapping classes of the genus two torus. Let $\Sigma_{2}$ be the closed orientable genus two surface, and let $M\left(\Sigma_{2}\right)$ be its mapping class group. There is a $2-$ fold branched covering $\pi: \Sigma_{2} \rightarrow S^{2}$; it has six branch points which we will denote by $y_{1}, \ldots, y_{6} \in S^{2}$. The group of covering transformations is generated by a hyper-elliptic involution $i$, and $[i]$ is central in $M\left(\Sigma_{2}\right)$. Let $M\left(S^{2}, 6\right)$ be the mapping class group of the sphere, with the branch points as the specified points.

Lemma 2.2 (Birman and Hilden [1, pages 183-189]) There is an exact sequence

$$
1 \rightarrow\langle[i]\rangle \rightarrow M\left(\Sigma_{2}\right) \stackrel{p}{\rightarrow} M\left(S^{2}, 6\right) \rightarrow 1
$$

where $p$ takes the standard generating Dehn twists $\left[\Delta_{1}\right], \ldots,\left[\Delta_{5}\right]$ to the standard generators of $M\left(S^{2}, 6\right),\left[\sigma_{1}\right], \ldots,\left[\sigma_{5}\right]$, which switch adjacent branch points on $S^{2}$. 
Definition 2.3 The Brunnian subgroup $\operatorname{Br}\left(\Sigma_{2}\right)$ of $M\left(\Sigma_{2}\right)$ is the preimage of the six strand Brunnian subgroup of the sphere:

$$
\operatorname{Br}\left(\Sigma_{2}\right)=p^{-1}\left(B r\left(S^{2}, 6\right)\right)
$$

Elements of $\operatorname{Br}\left(\Sigma_{2}\right)$ will be called Brunnian mapping classes of $\Sigma_{2}$. Note that $\operatorname{Br}\left(\Sigma_{2}\right)$ is a normal subgroup of $M\left(\Sigma_{2}\right)$.

Finally, we discuss homology with $\mathbb{Z}_{3}$ coefficients. Since $H_{1}\left(\Sigma_{2}, \mathbb{Z}_{3}\right)=\left(\mathbb{Z}_{3}\right)^{4}$, there is a representation

$$
\rho: M\left(\Sigma_{2}\right) \rightarrow S p\left(4, \mathbb{Z}_{3}\right)
$$

taking a mapping class to its induced map on homology.

We now restate the main theorem of this paper.

Theorem 1.2 The intersection of the Brunnian subgroup $\operatorname{Br}\left(\Sigma_{2}\right)$ and the kernel of the $\mathbb{Z}_{3}$ homology representation, $\rho: M\left(\Sigma_{2}\right) \rightarrow S p\left(4, \mathbb{Z}_{3}\right)$, is a nontrivial normal subgroup of $M\left(\Sigma_{2}\right)$, all of whose nontrivial elements are pseudo-Anosov.

Remark The methods used in this paper will not work to produce normal all pseudo-Anosov subgroups for higher genus surfaces, since those mapping class groups contain no central element to act as a covering transformation. However, we conjecture that normal all pseudo-Anosov subgroups do exist for higher genus surfaces.

\section{Preliminary lemmas}

We need the following fairly basic lemma, whose proof we include for the sake of completeness.

Lemma 3.1 Given a closed orientable surface $X$ with specified points $x_{1}, \ldots, x_{n}$, and a closed disk $D \subset X$ containing $x_{n}$ but none of $x_{1}, \ldots, x_{n-1}$, the map

$$
M_{p}(\overline{X-D}, n-1) \rightarrow M_{p}(X, n)
$$

induced by inclusion is an isomorphism.

Proof Recall that $M_{p}(\overline{X-D}, n-1)$ is defined to be the group of isotopy classes of self homeomorphisms of $\overline{X-D}$ that fix each of $x_{1}, \ldots, x_{n-1}$. Any homeomorphism or isotopy of $\overline{X-D}$ fixing $x_{1}, \ldots, x_{n-1}$ can be extended to 
one on $X$ fixing $x_{1}, \ldots, x_{n}$. To prove that the above map is one-to-one, we must show: given a homeomorphism $f: X \rightarrow X$ that fixes each of $x_{1}, \ldots, x_{n-1}$ and leaves $D$ invariant, and an isotopy $h_{t}: X \rightarrow X$ fixing $x_{1}, \ldots, x_{n}$ and going from the identity to $f$, there is an isotopy $H_{t}: \overline{X-D} \rightarrow \overline{X-D}$ going from the identity on $\overline{X-D}$ to the restriction of $f$ to $\overline{X-D}$.

Choose a closed disk $D^{\prime}$ such that $D \subset \operatorname{int} D^{\prime}, x_{1}, \ldots, x_{n-1} \notin D^{\prime}$, and further $h_{t}\left(\overline{X-D^{\prime}}\right) \cap D=\phi$ for all $t$. Let $g_{t}: D \cup \overline{X-D^{\prime}} \rightarrow X$ be an isotopy that is constant on $D$ and agrees with $h_{t}$ on $\overline{X-D^{\prime}}$. Using an isotopy extension theorem for topological manifolds [3], $g_{t}$ can be extended to an isotopy $G_{t}: X \rightarrow X . G_{0}$ is the identity map; thus by construction, $\left.G_{1}\right|_{X-D}$ is trivial in $M_{p}(\overline{X-D}, n-1)$.

Unfortunately, $\left.G_{1}\right|_{\overline{X-D}}$ may not agree with $\left.f\right|_{\overline{X-D}}$. They are the same on $\overline{X-D^{\prime}}$, but $\left.f\right|_{\overline{D^{\prime}-D}}$ may not equal $\left.G_{1}\right|_{\overline{D^{\prime}-D}}$. However, on $\overline{D^{\prime}-D}$, an annulus, any two functions are isotopic to each other by an isotopy which fixes $\partial D^{\prime}$ pointwise but can vary along $\partial D$. If we attach such an isotopy between $\left.G_{1}\right|_{\overline{D^{\prime}-D}}$ and $\left.f\right|_{\overline{D^{\prime}-D}}$ to the constant isotopy on $\overline{X-D^{\prime}}$, we get an isotopy $J_{t}: \overline{X-D} \rightarrow$ $\overline{X-D}$ going from $\left.G_{1}\right|_{\overline{X-D}}$ to $\left.f\right|_{\overline{X-D}}$.

Lemma 3.2 (Birman [2, page 217]) Let $X$ be a a closed orientable surface with specified points $x_{1}, \ldots, x_{n}$, and let $L_{n}: M_{p}(X, n) \rightarrow M_{p}(X, n-1)$ be the "forget $x_{n}$ map". Then there is a long exact sequence ending

$\rightarrow \pi_{1}\left(F_{p}(X, n-1)\right) \rightarrow \pi_{1}\left(X \backslash\left\{x_{1}, \ldots, x_{n-1}\right\}\right) \stackrel{d}{\rightarrow} M_{p}(X, n) \stackrel{L_{n}}{\rightarrow} M_{p}(X, n-1) \rightarrow 1$.

Moreover, ker $d$ is contained in the center of $\pi_{1}\left(X \backslash\left\{x_{1}, \ldots, x_{n-1}\right\}\right)$.

Remark Since $\pi_{1}\left(X \backslash\left\{x_{1}, \ldots, x_{n-1}\right\}\right)$ has trivial center unless $X=S^{2}$ and $n=3$, or $X=T$ and $n=1$, in all but these cases we get a short exact sequence

$$
1 \rightarrow \pi_{1}\left(X \backslash\left\{x_{1}, \ldots, x_{n-1}\right\}\right) \rightarrow M_{p}(X, n) \stackrel{L_{n}}{\rightarrow} M_{p}(X, n-1) \rightarrow 1 .
$$

Proof The proof follows the lines of Lemmas 4.1.1 and 4.2.1 in [B]. Namely, first one must show that the evaluation map

$$
\epsilon: F_{p}(X, n-1) \rightarrow X \backslash\left\{x_{1}, \ldots, x_{n-1}\right\}
$$

taking $h$ to $h\left(x_{n}\right)$ is a locally trivial fibering map with fiber $F_{p}(X, n)$. The exact sequence above is then the exact homotopy sequence of the fibering. Finally, elements of ker $d$ can be shown to commute with everything in $\pi_{1}\left(X \backslash\left\{x_{1}, \ldots, x_{n-1}\right\}\right)$. 


\section{Periodic and reducible Brunnian mapping classes of $S^{2}$ are trivial}

We first consider periodic maps in $M\left(S^{2}, n\right)$. Lemma 3.2 tells us that for $n \geq 4$, there is an exact sequence

$$
1 \rightarrow \pi_{1}\left(S^{2} \backslash\left\{x_{1}, \ldots, \widehat{x_{i}}, \ldots, x_{n}\right\}\right) \rightarrow M_{p}\left(S^{2}, n\right) \stackrel{L_{i}}{\rightarrow} M_{p}\left(S^{2}, n-1\right) \rightarrow 1
$$

for each $L_{i}$, the "forget about $y_{i}$ " map. Therefore, when $n \geq 4$, the subgroup of Brunnian sphere mapping classes is a free group, and has no nontrivial periodic elements.

Definition 4.1 A reducible homeomorphism in $F\left(S^{2}, n\right)$ is one that leaves invariant a collection of disjoint essential closed curves, none of which is isotopic to a simple loop around a single point. A reducible element of $M\left(S^{2}, n\right)$ is one with a reducible representative.

Theorem 4.1 For $n \geq 5$, an element of $\operatorname{Br}\left(S^{2}, n\right)$ that is also reducible must be trivial.

Proof Let $[g]$ be a reducible element of $\operatorname{Br}\left(S^{2}, n\right)$, for some $n \geq 5$, and let $g$ be a representative of $[g]$ that leaves invariant a collection of disjoint essential closed curves in $S^{2}$. Let $E$ be one these curves. $E$ separates the sphere into two open hemispheres, each containing at least two of the $n$ points $y_{1}, \ldots, y_{n}$. Some power $h=g^{r}$ leaves the curve $E$ itself invariant. Since $[h]$ is Brunnian, it does not permute the $n$ points, and so we can isotope $h$ so that that it restricts to the identity on $E$. We will show that $[h]$ is trivial. Then, since $\operatorname{Br}\left(S^{2}, n\right)$ contains no nontrivial periodic elements, $[g]$ must also be trivial.

Let $D^{+}$and $D^{-}$be the closures of the two components of $S^{2} \backslash E$. We will refer to these as the upper and lower hemispheres, respectively. If a homeomorphism $h$ fixes $E$ and the $n$ points $y_{1}, \ldots, y_{n}$, then it restricts to homeomorphisms of $D^{+}$and $D^{-}$. For convenience, we define the following notation: if $h \mid D^{+}=f$ and $h \mid D^{-}=g$, we write $h=(f, g)$. Similarly, given any two homeomorphisms $f$ and $g$ on the hemispheres that fix the points and $E,(f, g)$ denotes the homeomorphism of the sphere gotten by attaching $f$ and $g$ along $E$.

As we are interested in Brunnian mapping classes, let us consider what happens when we "forget" all but one of the points on the top hemisphere. Let $k$ be the number of the $y_{i}$ on the bottom hemisphere, so that there are $n-k$ points on the top hemisphere. Forgetting all but one of the $y_{i}$ from the top hemisphere will leave a total of $k+1$ points to be fixed under isotopies. 
Consider a Brunnian mapping class $[h]$ with a representative $h$ that fixes $E$. When we forget all but one point of the upper hemisphere, the image of $[h]$ in $M_{p}\left(S^{2}, k+1\right)$ must be trivial. Let $h_{+}=h \mid D^{+}$and $h_{-}=h \mid D^{-}$, so that $h=\left(h_{+}, h_{-}\right)$.

Claim $1\left[\left(h_{+}, h_{-}\right)\right]=\left[\left(1, h_{-}\right)\right]$as elements of $M_{p}\left(S^{2}, k+1\right)$.

Proof $M^{\partial}\left(D^{+}, 1\right)=1$, so there is an isotopy of the disk $D^{+}$, fixing the one point and the boundary, taking $h_{+}$to the identity. Attach this isotopy to the constant isotopy on $D^{-}$.

Claim 2 The homomorphism $j: M_{p}^{\partial}\left(D^{-}, k\right) \rightarrow M_{p}\left(S^{2}, k+1\right)$ taking $[f] \rightarrow$ $[(1, f)]$ has kernel generated by a single Dehn twist $\left[T_{\partial D^{-}}\right]$along a simple closed curve parallel to the boundary.

Proof $M_{p}\left(S^{2}, k+1\right) \cong M_{p}\left(D^{-}, k\right)$, by Lemma 3.1. However, the kernel of the map $M_{p}^{\partial}\left(D^{-}, k\right) \rightarrow M_{p}\left(D^{-}, k\right)$ is generated by the Dehn twist along a curve parallel to the boundary of $D^{-}$.

Since $\left[\left(1, h_{-}\right)\right]=\left[\left(h_{+}, h_{-}\right)\right]=[h]=1$ as elements of $M_{p}\left(S^{2}, k+1\right),\left[h_{-}\right]$is a power of $\left[T_{\partial D^{-}}\right]$in $M_{p}^{\partial}\left(D^{-}, k\right)$, and so $h_{-}$is isotopic to a power of $T_{\partial D^{-}}$by an isotopy fixing the boundary and all of the original $y_{i}$ that were on $D^{-}$.

Since we were careful to choose $E$ so that at least two points were on each hemisphere, we can repeat this argument upside down to show that $h_{+}$is isotopic to power of $T_{\partial D^{+}}$by an isotopy fixing the boundary $\partial D^{+}$and all of the original $y_{i}$ on $D^{+}$. By joining these two isotopies of the hemispheres along $E=\partial D^{+}=\partial D^{-}$, we acquire a isotopy on the sphere fixing all $n$ points. Thus

$$
[h]=\left[\left(h_{+}, h_{-}\right)\right]=\left[\left(T_{\partial D^{+}}^{r}, T_{\partial D^{-}}^{s}\right)\right]=\left[T_{E}^{r+s}\right]
$$

in $M_{p}\left(S^{2}, n\right)$, where $T_{E}$ is a Dehn twist along $E$, and $r$ and $s$ are integers.

To finish the proof of the lemma, we note that $M_{p}\left(S^{2}, 4\right)$ is a free group; if we forget all but two points on each hemisphere, $\left[T_{E}\right]$ is mapped to a generator of $M_{p}\left(S^{2}, 4\right)$, and thus $r+s=0$. This is where we need that $n \geq 5$, so that there is point to be lost to obtain a trivial element of $M_{p}\left(S^{2}, 4\right)$.

We have shown that there are no nontrivial periodic or reducible elements in $\operatorname{Br}\left(S^{2}, n\right)$ for $n \geq 5$. Thus all nontrivial elements must be pseudo-Anosov. 


\section{Reducible and periodic mapping classes on the genus two surface}

We now consider what happens to mapping classes of $\Sigma_{2}$ when they are projected to the sphere. Recall from section two that there is a branched covering $\pi: \Sigma_{2} \rightarrow S^{2}$ with branch points $y_{1}, \ldots, y_{6}$, and that we have chosen these points to be the six specified points for $M\left(S^{2}, 6\right)$. Also recall from Lemma 2.2 that there is a surjective map from $M\left(\Sigma_{2}\right)$ to $M\left(S^{2}, 6\right)$ whose kernel is generated by $[i]$, the mapping class of the covering involution. The Brunnian subgroup $\operatorname{Br}\left(\Sigma_{2}\right)$ is the preimage of $\operatorname{Br}\left(S^{2}, 6\right)$ under this map.

Any periodic element of $\operatorname{Br}\left(\Sigma_{2}\right)$ projects to a periodic element of $\operatorname{Br}\left(S^{2}, 6\right)$, which must be trivial. Thus, the only periodic element in $\operatorname{Br}\left(\Sigma_{2}\right)$ is the involution $[i]$.

Theorem 5.1 Reducible mapping classes in $M\left(\Sigma_{2}\right)$ project to reducible mapping classes in $M\left(S^{2}, 6\right)$.

Let $x_{1}, \ldots, x_{6} \in \Sigma_{2}$ be the preimages of the six branch points $y_{1}, . ., y_{6} \in S^{2}$. Let $C$ be a simple closed curve that is invariant under the involution $i$ and contains $x_{1}$ and $x_{2}$. $C$ will project down to a simple line segment connecting $y_{1}$ and $y_{2}$ on $S^{2}$. Let $C^{\prime}$ on $S^{2}$ be a simple closed curve that is the boundary of a small connected neighborhood of that line segment. See Figure 2.

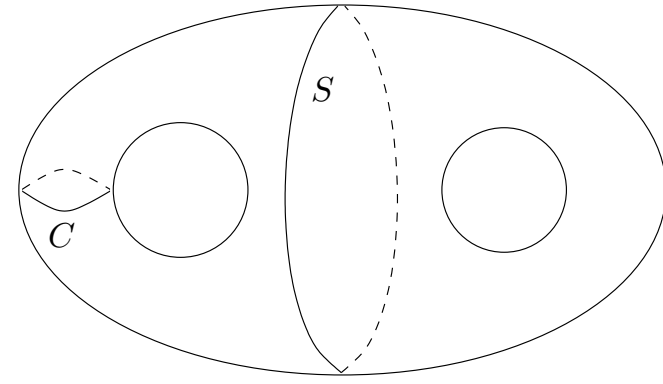

(a)

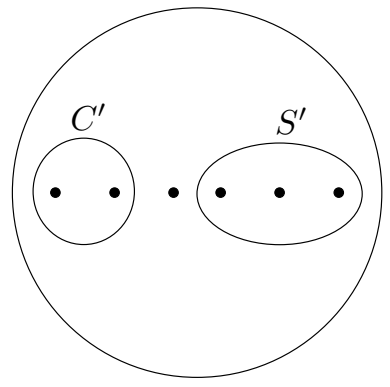

(b)

Figure 2: (a) Example of $S$ and $C$

(b) Example of $S^{\prime}$ and $C^{\prime}$

Let $S$ be an essential separating simple closed curve that is also invariant under $i$, does not intersect $C$, and which separates the branch cover points into sets $\left\{x_{1}, x_{2}, x_{3}\right\}$ and $\left\{x_{4}, x_{5}, x_{6}\right\}$. Let $S^{\prime}$ on $S^{2}$ be the image of $S$.

Note that although there are many choices for $S$ and $C$, these choices differ only by the action of elements of $M\left(\Sigma_{2}\right)$. 
Lemma 5.2 If $f: \Sigma_{2} \rightarrow \Sigma_{2}$ is a homeomorphism that leaves $C$ invariant, then $p([f])$ has a representative fixing $C^{\prime}$.

Proof The proof is to analyze what happens when we cut $\Sigma_{2}$ along $C$ and then cap off one of the holes.

Let $N(C)$ be an annular neighborhood of $C$. Then the closure of $\Sigma_{2} \backslash N(C)$ is a genus one surface with two disjoint disks removed. We will call this surface $T-2$. Recall that $M^{\partial}(T-2)$ is the group of isotopy classes of the set of homeomorphisms of $T-2$ that fix the boundaries pointwise. Inclusion of $T-2$ into $\Sigma_{2}$ induces a well defined map

$$
\phi: M^{\partial}(T-2) \rightarrow M\left(\Sigma_{2}\right) .
$$

Given an orientation preserving homeomorphism $f$ that leaves $C$ invariant, we can assume that $f$ or $f i$ restricts to the identity on the annular neighborhood $N(C)$. Since we need only prove a fact about $p([f])$, assume that $f$ fixes the annular neighborhood. Then $f$ restricts to a homeomorphism $f^{\prime}: T-2 \rightarrow T-2$ that fixes the boundary components pointwise. Thus $\left[f^{\prime}\right]$ is an element of $M^{\partial}(T-2)$, and $\phi\left(\left[f^{\prime}\right]\right)=[f]$; ie, $[f]$ is in the image of $\phi$. To prove the lemma it is enough to find generators for $M^{\partial}(T-2)$ and show that their images in $M\left(\Sigma_{2}\right)$ satisfy the conclusion.

Recall that we define $M(T-2)$ to be the group of isotopy classes of the set of homeomorphisms of $T-2$ that may vary along the boundary components, but do not switch them. There is an exact sequence

$$
1 \rightarrow\left\langle T_{\partial 1}, T_{\partial 2}\right\rangle \rightarrow M^{\partial}(T-2) \rightarrow M(T-2) \rightarrow 1
$$

where $T_{\partial 1}$ and $T_{\partial 2}$ are the twists about the boundaries.

If we cap off the holes of $T-2$ with pointed disks, we obtain a genus one torus $T$ with two specified points, $p_{1}$ and $p_{2}$. Recall that $M_{p}(T, 2)$ is the group of isotopy classes of the set of homeomorphisms of $T$ that fix both $p_{1}$ and $p_{2}$. Lemma 3.1 tells us that the homomorphism from $M(T-2)$ to $M_{p}(T, 2)$ induced by the capping off will be an isomorphism.

$$
M(T-2) \cong M_{p}(T, 2)
$$

If $L_{2}: M_{p}(T, 2) \rightarrow M(T, 1)$ is the "forget $p_{2}$ " map, then by the Remark after Lemma 3.2, the following sequence is exact.

$$
1 \rightarrow \pi_{1}\left(T-p_{1}\right) \rightarrow M_{p}(T, 2) \stackrel{L_{2}}{\rightarrow} M(T, 1) \rightarrow 1
$$


Definition 5.1 The image of a standard generator of $\pi_{1}\left(T-p_{1}\right)$ in $M_{p}(T, 2)$ is a double Dehn twist, that is, a pair of Dehn twists in opposite directions on curves immediately to either side of some simple closed curve representing the generator [5].

Each of these double Dehn twists is isotopic to the identity by an isotopy which fixes $p_{1}$ but lets $p_{2}$ move about; the tracks of $p_{2}$ under these isotopies represent the corresponding elements of $\pi_{1}\left(T-p_{1}\right)$.

If we let $a$ and $b$ be the standard generators for $\pi_{1}\left(T-p_{1}\right)$, then we define $\left[T T_{a}\right]$ and $\left[T T_{b}\right]$ as the images of $a$ and $b$.

$M(T, 1)$ is generated by Dehn twists $\left[T_{m}\right]$ and $\left[T_{l}\right]$ along the meridian and longitude of the punctured torus. Thus $M_{p}(T, 2)$ is generated by $\left[T T_{a}\right],\left[T T_{b}\right],\left[T_{m}\right]$, and $\left[T_{l}\right]$. See Figure 3. We will denote the preimages of these isotopy classes in $M^{\partial}(T-2)$ by $\left[\widehat{T T}_{a}\right],\left[\widehat{T T}_{b}\right],\left[\widehat{T}_{m}\right]$, and $\left[\widehat{T}_{l}\right]$. These, and $\left[T_{\partial 1}\right]$ and $\left[T_{\partial 2}\right]$, generate $M^{\partial}(T-2)$.

We can easily see that the images of these mapping classes in $M\left(\Sigma_{2}\right)$ all project down to mapping classes of the sphere with representatives that fix $C^{\prime}$.

Lemma 5.3 If $f: \Sigma_{2} \rightarrow \Sigma_{2}$ is a homeomorphism that leaves $S$ invariant, then $p\left([f]^{2}\right)$ has a representative fixing $S^{\prime}$.

Proof Again, we cut along $S$ and analyze the result.

Let $T_{1}-1$ and $T_{2}-1$ be the closures of the components of $\Sigma_{2} \backslash S$. Then the mapping class groups $M^{\partial}\left(T_{1}-1\right)$ and $M^{\partial}\left(T_{2}-1\right)$ are generated by Dehn twists along their meridians and longitudes and by Dehn twists along their boundaries. Again, there is a well defined map

$$
\phi: M^{\partial}\left(T_{1}-1\right) \times M^{\partial}\left(T_{2}-1\right) \rightarrow M\left(\Sigma_{2}\right),
$$

and the images of the generators project down to isotopy classes in $M\left(S^{2}, 6\right)$ that have representatives fixing $S^{\prime}$.

Given a homeomorphism $f$ that leaves $S$ invariant, $f$ either switches the two components of $\Sigma_{2}-S$, or it does not. In either case, $f^{2}$ cannot switch the two components of $\Sigma_{2}-S$. Thus we can assume that $f^{2}$ fixes an annular neighborhood of $S$. Therefore $f^{2}$ induces maps $f^{\prime}$ and $f^{\prime \prime}$ on $T_{1}-1$ and $T_{2}-1$ which fix the boundary, and hence $\left[f^{\prime}\right] \in M^{\partial}\left(T_{1}-1\right)$ and $\left[f^{\prime \prime}\right] \in M^{\partial}\left(T_{2}-1\right)$. But then $[f]^{2}=\phi\left(\left[f^{\prime}\right] \times\left[f^{\prime \prime}\right]\right)$. 

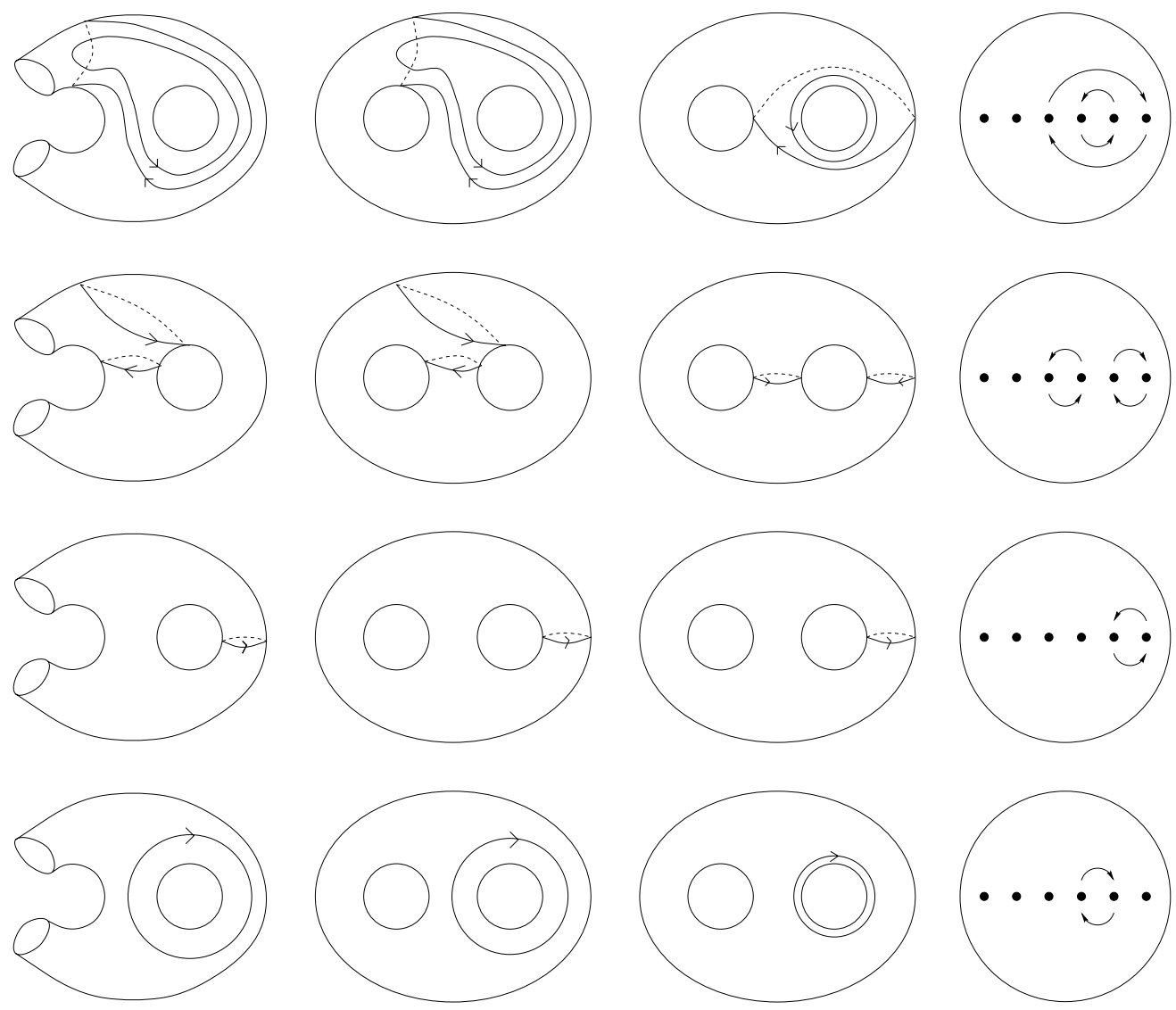

Figure 3: The four maps $\left[T T_{a}\right],\left[T T_{b}\right],\left[T_{m}\right]$, and $\left[T_{l}\right]$, and their images in $M\left(\Sigma_{2}\right)$ and $M\left(S^{2}, 6\right)$

Proof of Theorem 5.1 Let $[f] \in M\left(\Sigma_{2}\right)$ be a reducible mapping class. Then $[f]$ is conjugate to a mapping class $[g]$ which must fix one of the following collections of nonintersecting simple closed curves:

(1) $S$

(2) $S$ and $C$

(3) $S, C$, and another essential non-separating curve

(4) $C$

(5) $C$ and one other essential non-separating curve

(6) $C$ and two other essential non-separating curves 
In cases 1,2 , and $3, S$ must be left invariant, since it is the only separating curve. Therefore $p\left([g]^{2}\right)$ has a representative leaving $S^{\prime}$ invariant, by Lemma 5.3. In cases 4,5 , and 6 , there is some nonzero power $k$ so that $g^{k}$ has a representative leaving $C$ invariant. But then $p\left([g]^{k}\right)$ leaves $C^{\prime}$ invariant, by Lemma 5.2. Nonzero powers of reducible, periodic, and pseudo-Anosov elements are reducible, periodic, and pseudo-Anosov, repsectively. Therefore, by the classification theorem, in all cases above $p[g]$ is reducible, and so is $p[f]$.

We have shown that reducible elements of $M\left(\Sigma_{2}\right)$ project to reducible elements of $M\left(S^{2}, 6\right)$. Therefore, since $\operatorname{Br}\left(S^{2}, 6\right)$ has no nontrivial reducible elements, $\operatorname{Br}\left(\Sigma_{2}\right)$ also has no nontrivial reducible elements except the involution $[i]$.

\section{Nontriviality}

In Section 4 we proved that $\operatorname{Br}\left(S^{2}, n\right)$ has no nontrivial periodic or reducible elements. Thus, all of its nontrivial elements are pseudo-Anosov. In Section 5 , we proved that the only nontrivial element of $\operatorname{Br}\left(\Sigma_{2}\right)$ that is not pseudoAnosov is the involution $[i]$. To rid ourselves of this element, we intersect with the kernel of the usual $\mathbb{Z}_{3}$ homology representation, $\rho: M\left(\Sigma_{2}\right) \rightarrow S p\left(4, \mathbb{Z}_{3}\right)$.

Theorem 6.1 (Long [7, page 83]) The intersection of two nontrivial, noncentral normal subgroups of a mapping class group of a surface is nontrivial.

Since $\operatorname{Br}\left(\Sigma_{2}\right)$ and ker $\rho$ are not central, their intersection in $M\left(\Sigma_{2}\right)$ is nontrivial. As an example of a nontrivial element of $\operatorname{Br}\left(\Sigma_{2}\right) \cap \operatorname{ker} \rho$, consider the nested commutator

$$
\left[\Delta_{1}^{6},\left[\Delta_{2}^{6},\left[\Delta_{3}^{6},\left[\Delta_{4}^{6}, \Delta_{5}^{6}\right]\right]\right]\right],
$$

where $\Delta_{i}$ are the standard generating Dehn twists on $\Sigma_{2}$. This mapping class is Brunnian, since it projects to

$$
\left[\sigma_{1}^{6},\left[\sigma_{2}^{6},\left[\sigma_{3}^{6},\left[\sigma_{4}^{6}, \sigma_{5}^{6}\right]\right]\right]\right],
$$

which clearly reduces to the identity if any of the six points on the sphere is forgotten. It also acts trivially on homology with $\mathbb{Z}_{3}$ coefficients, but it is not null-homologous over $\mathbb{Z}$, and so it is not trivial.

Other examples can be easily generated by taking commutators of Brunnian mapping classes and null- $\mathbb{Z}_{3}$-homologous elements, or simply by using homology to check that a given Brunnian mapping class is not $[i]$. This provides a new source of pseudo-Anosov mapping classes of the genus two torus. 
Acknowledgments This paper is part of my dissertation. I would like to thank John Stallings for serving as my thesis advisor, and Andrew Casson for numerous conversations and suggestions. I would also like to thank the referees. This research was partly funded by NSF grant DMS 950-3034.

\section{References}

[1] J Birman, Braids, Links, and Mapping Class groups, Annals of Math. Studies, No. 82. Princeton University Press

[2] J Birman, Mapping class groups and their relationship to braid groups, Comm. Pure and Applied Math. 22 (1969) 213-238

[3] R Edwards, R Kirby, Deformations of spaces of imbeddings, Annals of Math. 98 (1971) 63-88

[4] Fathi, F Laudenbach, V Ponerau, et al, Travaux de Thurston sur les surfaces, Astérisque 66-67 (1979)

[5] D Johnson, Homeomorphsims of a surface which act trivially on homology, Proc. of AMS, 75 (1979) 119-125

[6] H Levinson, Decomposable braids and linkages, Trans. AMS, 178 (1973) 111126

[7] D Long, A note on the normal subgroups of mapping class groups Math. Proc. Camb. Phil. Soc. 99 (1985) 79-87

[8] D Rolfsen, Knots and Links, Publish or Perish, Inc. Math. Lect. Series 7 (1976) 